\title{
Emission of Diffuse Bands of Sodium behind Shock Fronts*
}

\author{
H. Takeyama $\nmid$ and A. GutTman $\neq$ \\ Daniel and Florence Guggenheim Jet Propulsion Center, \\ California Institute of Technology, Pasadena, California
}

(Received October 14, 1959)

W $\mathrm{URM}^{1}$ has described a continuous emission band extending from $5490 \mathrm{~A}$ to the $D$ lines and inferred the existence of van der Waals states of $\mathrm{Na}_{2}$. Hamada ${ }^{2}$ has classified sodium bands in the red and yellow regions into three groups, viz., (1) red bands ( $\lambda \lambda 8150-$ $5500)$, (2) cathode ray fluorescence band, and (3) the continuous band $(\lambda \lambda 5470-7200)$. Recently, wide diffuse bands have been observed and discussed qualitatively for rubidium-rare gas mixtures. ${ }^{3,4}$

By utilizing the shock tube, we have observed the emission of a continuous band corresponding to $\mathrm{Ha}-$ mada's type (3) band. We used finely ground salts as a source of sodium in a one-inch diameter tube; the lowpressure section was $51 \mathrm{in.} \mathrm{long} \mathrm{and} \mathrm{the} \mathrm{high-pressure}$ section had a length of 24 in. Mylar diaphragms $(0.003$ and $0.005 \mathrm{in}$. thick) separated the two sections. Observations were made through a lucite block at the end of the low pressure section. Spectrograms were taken with a $1.5-\mathrm{m}$ ARL spectrograph with a dispersion of $6.8 \mathrm{~A} / \mathrm{mm}$. Kodak Royal-X panchromatic sheet film and Kodak Tri-X panchromatic roll film were used. Intensity measurements were made with an ARL 2250 densitometer.

Measured quantities (from 5 to $300 \mathrm{mg}$ ) of finely ground sodium salts (e.g., $\mathrm{NaCl}, \mathrm{NaBr}$, and $\mathrm{Na}_{2} \mathrm{CO}_{3}$ ) were placed in the Lucite block and spread evenly. Argon, at pressures from 5 to $100 \mathrm{~mm} \mathrm{Hg}$, was admitted to the low-pressure section. The high-pressure section was filled with helium and a magnetic breaker used to burst the diaphragm.

We observed atomic lines of $\mathrm{Na}$ and a continuous band under various shock conditions. As the amount of sodium salt is increased at constant shock conditions, the $D$ lines become stronger, wider and self-reversed; also the diffuse series lines $(\lambda \lambda 5682,5688)$ and sharp series lines $(\lambda \lambda 6154,6160)$ appear. When the quantity of sodium chloride exceeds about $10 \mathrm{mg}$, the diffuse band becomes superimposed on the atomic lines. Further increases in the amount of sodium chloride up to $300 \mathrm{mg}$ did not change the general features of the spectrum. A typical intensity profile (Fig. 1) shows a continuous band starting at about $5490 \mathrm{~A}$ and extending beyond $6200 \mathrm{~A}$. The quantities $T_{5}$ and $p_{5}$ were estimated by using ideal, one-dimensional shock theory. ${ }^{5}$ An intermediate maximum occurs at about $5500 \mathrm{~A}$; on the long-wavelength side the intensity decreases continuously without distinct cutoff.

There is little doubt that the continuous band $\lambda \lambda 5490-6200$ is identical with Hamada's (3) band and Wurm's band. The discrepancy in the long-wavelength

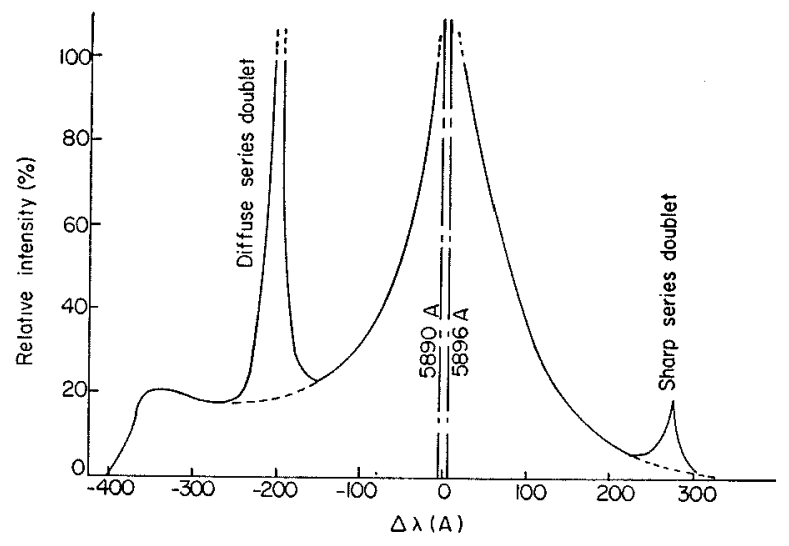

FIG. 1. Intensity distribution of band accompanying $D$ lines; $p_{1}=50 \mathrm{~mm} \mathrm{Hg}, p_{4}=395 \mathrm{psi}, p_{5}=12$ atmos, $T_{5}=6300^{\circ} \mathrm{K}, \mathrm{NaCl}=$ $50 \mathrm{mg}$.

extent of the band between Hamada's results and ours may be attributed to the extreme difference of excitation conditions. The principal support for the assertion that the band belongs to $\mathrm{Na}_{2}$ molecular states comes from the observation that the emission spectrum is independent of the nature of the foreign gas. Wurm considered the structure in the long-wavelength region of the $D$ lines to result from absorption of the red band of $\mathrm{Na}_{2}$, the background being the broadening of the $D$ lines. On the other hand, Hamada interpreted the entire continuum (both sides of the $D$ lines) to constitute a single band caused by the same mechanism described by Wurm. Recently, Ch'en, Bennett, and Jefimenko ${ }^{3}$ have presented potential curves that are consistent with both the red and violet bands. It appears reasonable to conclude that the continuous band results from a transition between a molecular state composed of ${ }^{2} S$ and ${ }^{2} P$ Na atoms and a similar molecule composed of two ground state $\mathrm{Na}$ atoms. It should be noted that $\mathrm{Na}_{2}$ molecules should be formed behind shock fronts after interaction between the incident or reflected shocks and the rarefaction wave.

Further research of a more quantitative nature is now in progress and will be reported in the near future.

* Supported by the U. S. Air Force, Office for Advanced Studies, under Contract AF 18(603)-2. The authors are indebted to Dr. S. S. Penner for helpful discussions.

$\dagger$ Senior Research Fellow 1958-1960; permanent address: Department of Physics, Hiroshima University, Hiroshima, Japan.

$\ddagger$ Guggenheim Fellow in Jet Propulsion, 1958-1959.

${ }^{1}$ K. Wurm, Z. Physik 79, 736 (1932).

${ }^{2}$ H. Hamada, Phil. Mag. 15, (7), 574 (1933).

${ }^{3}$ Ch'en, Bennett, and Jefimenko, J. Opt. Soc. Am. 46, 182 (1956).

${ }_{4}^{4} \mathrm{O}$. Jefimenko and W. Curtis, J. Chem. Phys. 27, 953 (1957).

${ }^{5}$ Ressler, Lin, and Kantrowitz, J. Appl. Phys. 23, 1390 (1952). 